Artículos

\title{
Efecto de la variable género en la disponibilidad léxica de estudiantes de inglés como lengua extranjera
}

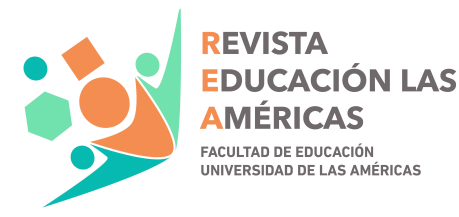

\author{
Effect of the gender variable on the lexical availability of \\ students of English as a foreign language
}

Quintanilla Espinoza, Angie Evelyn; Kloss Medina, Steffanie

D Angie Evelyn Quintanilla Espinoza

anquinta@udec.cl

Universidad de Concepción, Chile

(iD) Steffanie Kloss Medina

skloss@ucsc.cl

Universidad Católica de la Santísima Concepción, Chile

Revista Educación las Américas

Universidad de Las Américas, Chile

ISSN-e: 0719-7128

Periodicidad: Semestral

vol. 10, núm. 2, 2020

ccalisto@udla.cl

Recepción: 16 Noviembre 2020

Aprobación: 30 Diciembre 2020

URL: http://portal.amelica.org/ameli/ jatsRepo/248/2481629008/index.html

Cada autor garantiza que su trabajo enviado es original y que tiene todo el poder para celebrar este acuerdo. Ni este trabajo ni otro similar se han publicado en otro lugar en ningún idioma ni se enviarán para su publicación en otro lugar mientras REA los esté evaluando. El autor conserva sus derechos de autor y garantiza a la revista el derecho de la primera publicación de su obra.

\section{c) (i) (8)}

Esta obra está bajo una Licencia Creative Commons AtribuciónNoComercial 4.0 Internacional.
Resumen: La disponibilidad léxica se focaliza en el estudio del léxico de determinadas comunidades de hablantes, a partir del análisis de frecuencia de palabras. En este contexto, la presente investigación evalúa el efecto de la variable género en la disponibilidad léxica en inglés como lengua extranjera en una muestra de 46 estudiantes ( 24 mujeres y 22 hombres) de quinto año básico de un colegio de administración subvencionada. El objetivo de esta investigación es analizar cuantitativamente el efecto del factor género en la disponibilidad léxica en inglés en los centros de interés: Colores, miembros de la familia, deportes, animales y comidas y bebidas. Este análisis se realiza a partir de los siguientes índices: número de palabras $(\mathrm{N})$, promedio de palabras (XR), número de palabras diferentes (NPD) e índice de cohesión (IC). Los resultados obtenidos en la prueba de asociatividad muestran que, si bien ambos grupos disponen de un variado léxico disponible en inglés, la variable género no tendría incidencia a nivel cuantitativo, dado que no existen diferencias significativas en los resultados obtenidos en la prueba de disponibilidad léxica aplicada a los estudiantes.

Palabras clave: léxico, enseñanza de idiomas, enseñanza de una segunda lengua, lingüística.

Abstract: Lexical availability focuses on studying the lexicon of specific communities of speakers based on the analysis of the frequency of words. In this context, the present research evaluated the effect of the gender variable on lexical availability in English as a foreign language in a sample of 46 fifth-grade students ( 24 women and 22 men) of a subsidized school. This research aimed to quantitatively analyze the effect of the gender factor on lexical availability in English in the centers of interest: colors, family members, sports, animals, and food and drink. This analysis was conducted using the following indicators: number of words $(\mathrm{N})$, the average number of words (XR), the number of different words (NPD), and cohesion index (IC). The results obtained in the associativity test showed that, although both groups have a varied available lexicon in English, the gender variable would not impact a quantitative level since there are no significant differences in the lexical availability test.

Keywords: lexis, language teaching, second language teaching, linguistics. 


\section{INTRODUCCIÓN}

Los estudios en torno al aprendizaje de lenguas consideran que el aprendizaje léxico es un elemento clave, de este modo, algunos autores señalan que la falta de manejo léxico impediría una comunicación efectiva (Lightbown y Spada, 2013; Palapanidi, 2012). Asimismo, Palapanidi (2012) señala que la adquisición léxica es un proceso prolongado y constante que se relaciona con las experiencias que vive el aprendiz. En este mismo ámbito, Cepeda, Granada y Pomes (2014) plantean que los factores sociales que emergen a partir del contexto "son determinantes en la adquisición y uso de la lengua materna y sus diferencias según género, entre estos factores se destacan la influencia de los padres, la escuela, la televisión y las prácticas socioculturales” (p. 185). Al considerar que factores sociales como el género son determinantes en la adquisición de la lengua materna, es posible considerar que también podrían serlo en el proceso de adquisición de una lengua extranjera como lo señalan Jiménez y Ojeda (2009) y Agustín y Terrazas (2012).

Las investigaciones en disponibilidad léxica han evidenciado resultados contradictorios, algunos estudios muestran la superioridad léxica de las mujeres (Jiménez y Ojeda, 2009; Fernández, 2010; Calero y Serrano, 2019) evidencian la superioridad de los hombres (Galloso, 2003; Hernández, 2006), mientras que otro grupo de estudios propone que no existirían diferencias significativas en la disponibilidad léxica de hombres y mujeres (Cepeda et al., 2014; Pacheco, Cabrera y Gonzales, 2017).

La presente investigación tiene como propósito evaluar el efecto de la variable género en la disponibilidad léxica en inglés como lengua extranjera para establecer si existen diferencias entre el léxico disponible de hombres y mujeres. Este interés surge dado que la variable género ha sido ampliamente investigada en los estudios de disponibilidad léxica en lengua materna, sin embargo, son pocos los estudios que han considerado el efecto del género en una lengua extranjera, y aún menos los que lo han hecho en la lengua inglesa.

A fin de poder indagar en esta variable y establecer posibles diferencias entre hombres y mujeres, se cuantificará el léxico disponible de los sujetos a partir de los estadígrafos comúnmente utilizados en disponibilidad léxica, se compararán estos índices, se realizarán pruebas estadísticas y finalmente se efectuará una descripción de las 10 palabras más disponibles en hombres y mujeres.

El presente artículo está organizado en las siguientes secciones: sección 2 que desarrolla el marco teórico en torno a la disponibilidad léxica y los estudios en género, sección 3 que presenta el marco metodológico que guía el estudio, sección 4 que examina los resultados obtenidos y sección 5 que presenta las conclusiones de la investigación y discute los hallazgos.

\section{Marco Teórico}

\subsection{Disponibilidad Léxica}

Los primeros estudios en el área de disponibilidad léxica fueron realizados a mediados del siglo XX en Francia, impulsados por la elaboración del libro Francais élémentaire (Gougenheim, Michéa, Rivenc y Sauvageot, 1956), especialmente concebido con el propósito de "elaborar una "lengua base" a partir de una gramática y un léxico fundamental para enseñar francés a inmigrantes" (Quintanilla y Salcedo, 2019, p. 531).

En un inicio, el trabajo de los investigadores se centró en el estudio del léxico frecuente, sin embargo, este criterio no resultó apropiado dado que en estos listados no aparecían (o aparecían en posiciones retrasadas) palabras de uso cotidiano esenciales para la comunicación. Los resultados obtenidos en base al estudio del léxico frecuente no satisficieron las expectativas de los investigadores por lo cual determinaron utilizar pruebas de tipo asociativo (Michéa, 1953) "en las que se les pide a los hablantes que enuncien o escriban las palabras que asocian con una determinada temática o centro de interés” (Quintanilla y Salcedo, 2019, p.532). Estas pruebas asociativas debían estimular el lexicón mental de los hablantes para que de esta forma 
se pudiera producir palabras que estaban disponibles en el léxicon mental, pero que solo son activadas en instancias comunicativas específicas. De este modo, Michéa (1953) señala

Una palabra disponible es una palabra que, sin ser particularmente frecuente, siempre está lista para ser usada y se presenta de forma inmediata y natural a la mente en el momento en que se la necesita. Es una palabra que, formando parte de asociaciones de ideas usuales, existe en potencia en el hablante, en cuanto entran en juego dichas asociaciones (p.4).

$\mathrm{Al}$ respecto, Hernández y Tomé (2017) plantean que "cuando hablamos de léxico disponible estamos haciendo referencia a un conjunto de unidades léxicas que están altamente disponibles para un hablante de una lengua concreta cuando se le pregunta por una determinada categoría semántica” (p. 100). Otra definición de léxico disponible es la propuesta por Galdámez, Guerrero y Toledo (2018) quienes señalan que este léxico corresponde a "las palabras temáticas que aparecen según un campo semántico determinado y que están en el lexicón (no estático) del aprendiente, disponibles para ser empleadas según el contexto de uso" (p.139).

\subsection{Fórmula para el cálculo del índice de disponibilidad léxica}

El índice de disponibilidad léxica es un indicador que sirve para expresar el grado de disponibilidad de un término en la mente del hablante, esto es, la facilidad o dificultad con que un vocablo aflorará a la conciencia del sujeto en el momento de necesitarlo. Este índice está compuesto por tres factores:

$\mathrm{f}=$ Frecuencia de las menciones de la palabra.

$\mathrm{N}=$ Número de sujetos encuestados.

$\Lambda=$ Factor de ponderación.

El índice resultante será directamente proporcional a la frecuencia, es decir a las veces que el vocablo ha sido mencionado. Por otra parte, el $\mathrm{N}$ debe ser considerado, pues la probabilidad de ocurrencia del término es función de aquel. Por último, aparece Lambda, que establece el peso que le queremos dar a la posición que el término ocupe en la lista entregada por cada sujeto: a mayor distancia de la primera posición, el valor del índice de disponibilidad léxica (IDL) será más bajo. Esto se expresa elevando al cuadrado, al cubo, etc., según se aleja la ocurrencia del primer lugar. De este modo la frecuencia queda ponderada por la posición y dividida por N. La expresión formulada y que será utilizada en la investigación (ver figura 1), fue la diseñada por Lorán y López (1983).

$$
D(P)=\frac{f_{1}+\lambda f_{2}+\lambda^{2} f_{3}+\ldots .+\lambda^{n-1} f_{n}}{N}
$$

[Figura 1]

\subsection{Estudios en disponibilidad léxica en L2 y género}

El estudio de las diferencias sexolectales, en el área de disponibilidad léxica, ha estado presente desde sus inicios (Gougenheim et al.,1956). En este sentido, el interés principal ha sido determinar si existen diferencias (índices superiores) entre la producción léxica de hombres y mujeres. La mayoría de estos estudios se ha centrado en explorar esta variable en la lengua materna. Sin embargo, esta sección presenta estudios que consideran la variable género en la disponibilidad léxica en una lengua extranjera, en este caso en particular se presentan estudios en español y en inglés. 
Entre los estudios en español como lengua extranjera destacan los de Verdeses-Mirabal (2012), Sandu (2013) y Galdames, Guerrero y Toledo (2018). El estudio de Verdeses-Mirabal (2012) examinó el efecto de la variable género en léxico disponible en español de estudiantes californianos. Sus hallazgos evidencian que las mujeres evocaron una mayor cantidad de respuestas en cada uno de los índices de la prueba de DL y que la diferencia entre géneros es estadísticamente significativa. Este estudio indica que la variable género es el factor que tiene mayor incidencia en todos los índices, considerando otras variables estudiadas como la L1, el nivel de español o el nivel sociocultural.

El trabajo realizado por Sandu (2013) examina el efecto del género en la disponibilidad léxica y el nivel de escolaridad de una muestra de 280 estudiantes. Los resultados obtenidos muestran que las mujeres obtienen valores más altos en todos los índices de los 16 centros de interés incluidos en la prueba de DL, los cuales mostraron ser estadísticamente significativos.

El trabajo de Galdames et al. (2018) no presenta diferencias significativas a nivel cuantitativo entre el léxico de hombres y mujeres, y permitió establecer un léxico común para ambos sexos a nivel cualitativo.

Por su parte, entre los estudios en inglés como lengua extranjera destacan los de Jiménez y Ojeda (2009), Fernández, (2010) y Agustín y Fernández (2014). En la investigación efectuada por Jiménez y Ojeda (2009) en niños y niñas de sexto grado de educación primaria, las niñas mostraron una mayor productividad léxica que los niños en los 15 centros de interés en estudio. Los resultados permitieron concluir una diferencia significativa entre la cantidad de palabras producidas por niñas y niños, la cual es más relevante en algunos centros de interés, sin embargo, el mayor patrón de rendimiento de las niñas fue consistente en todos los centros en estudio.

Otro estudio que tuvo como objetivo observar las diferencias de género en la disponibilidad léxica en inglés como lengua extranjera fue el desarrollado por Fernández (2010). Este trabajo reveló diferencias significativas entre ambos sexos, mostrando una preponderancia en los resultados del género femenino.

Finalmente, Agustín-Llach y Fernández (2014) utilizó una muestra de 190 estudiantes de estudiantes de inglés como lengua extranjera para estudiar el efecto de la variable género a lo largo del tiempo y midió su DL en dos momentos (12 años y 15 años). Los datos obtenidos mostraron que las mujeres superaron a los hombres en las dos mediciones en 6 de los 9 centros de interés de la prueba de disponibilidad léxica. Los resultados mostraron un aumento de vocabulario en ambos grupos a lo largo del tiempo, sin embargo, las diferencias mostradas fueron estadísticamente significativas, favoreciendo a las mujeres por sobre los hombres.

\subsection{Estudios de disponibilidad léxica y género}

Como se mencionó al principio de esta sección, varios han sido los estudios que se han dedicado a explorar la variable género. A pesar de que los resultados obtenidos han sido contradictorios, en general se han observado los siguientes fenómenos:

a) las mujeres superan a los hombres (Jiménez y Ojeda, 2009; Trigo y González, 2011; Agustín y Fernández, 2014; Calero y Serrano, 2019, entre otros)

los hombres superan a las mujeres (Galloso, 2003; Hernández, 2006, entre otros)

no se observan diferencias significativas a nivel cuantitativo en el caudal léxico (Areta, 2009; Pacheco, Cabrera y Gonzales, 2017, entre otros).

Sin embargo, gran parte de los estudios en género y disponibilidad léxica concuerdan en que esta variable es la que menos influiría en el caudal léxico de los informantes, ya que, en general, no observan diferencias a nivel cuantitativo en el léxico de hombres y mujeres. No obstante, es posible identificar diferencias a nivel cualitativo, las que, de acuerdo con Pacheco et al. (2017) se atribuyen a: 
a) actividades o atributos considerados tradicionalmente como femeninos o masculinos en centros de interés que se refieren a dichas actividades (Trigo y González, 2011).

al orden en el cual se actualizan los vocablos y el tipo de términos incorporados (Sandu, 2013).

la naturaleza del léxico utilizado por las mujeres tiende a ser más más prestigioso socialmente y más conservador (González y Orellana ,1999).

\section{Metodología}

Esta investigación sigue un enfoque cuantitativo dado que persigue recolectar datos con el objetivo de contrastar una hipótesis, sobre la base de datos numéricos y análisis estadístico. En este caso en particular, se utilizan los siguientes índices:

- $\quad \mathrm{N}$ : número total de palabras evocadas por los individuos de la muestra frente a un centro de interés. XR: promedio de vocablos indicados por los individuos de la muestra frente a un centro de interés NPD: cantidad de palabras diferentes en el centro de interés, el cual considera cada palabra sólo una vez.

IC: grado de coincidencia en las respuestas de los individuos y corresponde a la razón XR/NPD.

Desde el punto de vista metodológico, este estudio se enmarca en una investigación de carácter descriptivo dado que se busca detallar el léxico disponible de un grupo de sujetos y describir las frecuencias, promedios e índices de cohesión de la prueba de disponibilidad léxica aplicada a los sujetos de la muestra.

En este mismo contexto, la investigación llevada a cabo posee un diseño no experimental, ya que los datos se recogen solo en un momento determinado, en un tiempo único y no existe manipulación de las variables de la investigación.

La muestra de este estudio está compuesta por 46 estudiantes de quinto año básico (entre 10 y 11 años) de un colegio de administración subvencionada chileno. La muestra está conformada por 24 mujeres y 22 hombres, los cuales han cursado la lengua extranjera inglés desde el nivel Pre-Kínder.

\subsection{Instrumento}

El instrumento utilizado en esta investigación es un test de disponibilidad léxica el cual refleja la producción espontánea de vocabulario por parte de los sujetos (Payne, 2016 p.18).

Este instrumento consideró los siguientes centros de interés: colors, family members, sports, animals y food and drink, los cuales fueron escogidos considerando las unidades que ya habían sido impartidas a estos alumnos de acuerdo con los planes y programas entregados por el Ministerio de Educación.

El test incluyó dos secciones, la primera de ellas recopila información de identificación del sujeto (edad y género), mientras que la segunda sección incorpora los 5 centros de interés en estudio para los cuales cada sujeto debe producir una lista de palabras en un tiempo de dos minutos por centro, siguiendo la corriente actual utilizada en los estudios de disponibilidad léxica (Garzón y Penagos, 2016).

Las instrucciones se entregaron de forma oral y se presentaron los centros de forma individual cada 2 minutos. El test tuvo una duración total de 15 minutos.

\subsection{Procesamiento de datos}

Una vez recopilados los datos, estos se separaron en dos grupos, hombres y mujeres. Luego, la información fue ingresada en un documento Excel para edición de los datos. Durante la edición de datos se validaron las respuestas y se procedió a limpiar el corpus (lematizado) a partir de las siguientes directrices: 
a) Se descartaron las pruebas que tenían un centro de interés en blanco.

Se descartaron las palabras en español y las que no aparecen como entradas léxicas en los diccionarios. (Oxford Learners Dictionary Online https://www.oxfordlearnersdictionaries.com/ )

Se descartaron las palabras repetidas en el mismo centro de interés.

Se corrigieron los errores de ortografía si se entendía el significado de la palabra.

Se consideraron formas singulares.

Se eliminaron las marcas comerciales.

Se editaron las palabras en mayúsculas.

Se separaron las palabras compuestas utilizando un guion.

Además, en esta planilla Excel, según se observa en la figura 2, se ingresó el número de cada uno de los encuestados para su identificación y el orden en que fueron escritos los vocablos dentro de cada centro de interés.

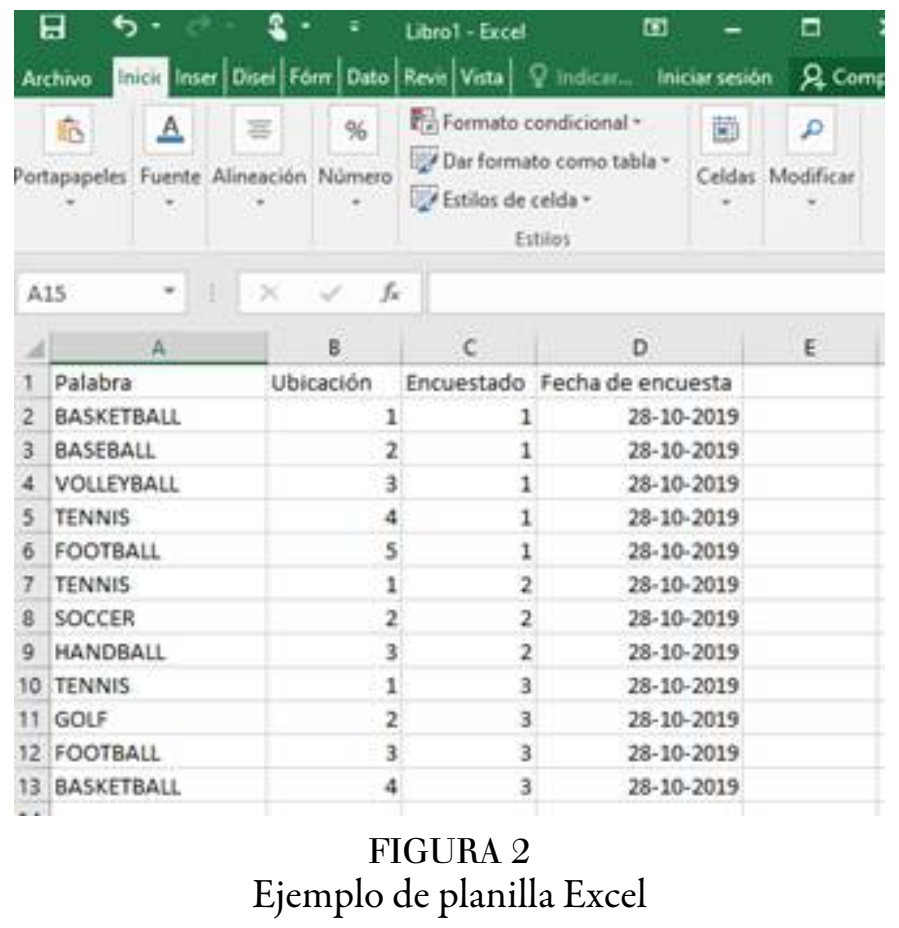

Posteriormente para generar datos concretos para el análisis, se ingresan dichos datos a la plataforma Lexmath para generar un reporte para cada centro de interés, el cual incluye el N, el XR, el NPD y el IC, como se aprecia en la figura 3. Finalmente, esta plataforma entrega un listado con las palabras que fueron utilizadas dentro de este grupo o centro de interés. 


\section{LexMath}

\section{Reporte.}

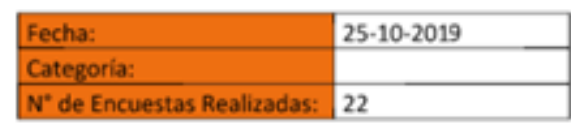

\begin{tabular}{|c|c|c|c|}
\hline Total de Palabras & XR & PD & IC \\
\hline 85 & 3.86 & 13 & 0.29720 \\
\hline
\end{tabular}

\begin{tabular}{|c|c|c|c|}
\hline Palabra & Frecuencia Absoluta & Frecuencia Relativa & IDL \\
\hline MOM & 19 & 0,86364 & 0,810863636 \\
\hline GRANDPA & 14 & 0,63636 & 0,521362727 \\
\hline DAD & 12 & 0,54545 & 0,501363636 \\
\hline BROTHER & 11 & 0,5 & 0,361824136 \\
\hline SISTER & 10 & 0,45455 & 0,334276364 \\
\hline GRANDMA & 5 & 0,22727 & 0,179145455 \\
\hline MOTHER & 3 & 0,13636 & 0,136363636 \\
\hline UNCLE & 3 & 0,13636 & 0,100554546 \\
\hline GRAND-FATHER & 2 & 0,09091 & 0,082272727 \\
\hline FATHER & 2 & 0,09091 & 0,081818182 \\
\hline
\end{tabular}

FIGURA 3

Reporte entregado por plataforma Lexmath

\section{Resultados}

El presente apartado da cuenta de los resultados obtenidos a partir de la prueba de disponibilidad léxica aplicada a los sujetos de la muestra. Este análisis se realizará presentando la disponibilidad léxica de mujeres y hombres en cuanto a los índices cuantitativos en estudio (N, XR, NPD e IC) en los 5 centros en estudio. Luego, se presentará un análisis estadístico de estos datos con el propósito de establecer si existen diferencias significativas en estos índices. Finalmente, se presentarán los datos cuantitativos de cada uno de los centros de manera individual, además de presentar una lista con las 10 palabras más disponibles en mujeres y hombres.

\subsection{Disponibilidad léxica en mujeres}

En la tabla 1 se detallan los resultados específicos de disponibilidad léxica para el subgrupo mujeres de la muestra de la investigación. Cabe señalar que el número de palabras es la sumatoria de las palabras correctamente escritas y que XR representa el promedio de palabras producido por cada alumna por categoría.

TABLA 1

Resultados de IDL para mujeres

\begin{tabular}{lllll}
\hline Centros de interés & N & XR & NPD & IC \\
Color's & 148 & 6.73 & 16 & 0.42045 \\
Animals & 146 & 6.35 & 24 & 0.26449 \\
Food and drink & 106 & 5.05 & 25 & 0.20190 \\
Family members & 85 & 3.86 & 13 & 0.29720 \\
Sports & 55 & 2.89 & 11 & 0.26316 \\
\hline
\end{tabular}

Frente a los datos recogidos en las mujeres, se puede observar que el centro de interés que presenta el mayor nivel es el centro colors, con un número total de palabras de 148 producidas por todas las alumnas de quinto, 
un promedio de palabras de 6.73 por alumna y un IC de 0.42045 . El centro de interés animals, presenta 146 palabras lo que evidencia un alto repertorio de palabras disponibles dentro de esta categoría. Por otro lado, los centros food and drink ( 106 palabras), family members ( 85 palabras) y sports ( 55 palabras), son los que presentaron un menor nivel de disponibilidad léxica al momento de realizar la evaluación.

\subsection{Disponibilidad léxica en hombres}

Considerando el número de palabras diferentes evocadas por los varones, en la tabla 2 se puede apreciar que la población masculina tuvo un mejor desempeño en los centros de interés animals y colors, con 166 y 161 palabras respectivamente. El promedio de palabras entregadas en esta categoría fluctúa entre 7.22 y 7.0 respectivamente.

TABLA 2

Resultados de IDL para hombres

\begin{tabular}{lllll}
\hline Centros de interés & N & XR & NPD & IC \\
\hline Animals & 166 & 7.22 & 28 & 0.25776 \\
Colors & 161 & 7.00 & 18 & 0.38889 \\
Food and drink & 115 & 5.48 & 26 & 0.21062 \\
Family members & 93 & 4.23 & 12 & 0.35227 \\
Sports & 83 & 3.61 & 11 & 0.32806 \\
\hline
\end{tabular}

Luego, en el caso de food and drink el número de palabras desciende a 115, producidas por todos los alumnos de quinto, con un promedio de 5.48 palabras, 1.74 palabras menos que el centro de interés animals. Finalmente, los centros family members y sports son los centros con menor número de palabras entregadas por los sujetos con 93 y 83 cada uno, descendiendo a promedios de palabras de 4.23 y 3.61 .

\subsection{Análisis estadístico}

Con el objetivo de conocer si existen diferencias significativas en el léxico disponible en inglés entre hombres y mujeres, se realizó una prueba t considerando los siguientes índices: número de palabras $(\mathrm{N})$, promedio de palabras (XR) y número de palabras diferentes (NPD).

a) número de palabras $(\mathrm{N})$ : El primer estadígrafo analizado corresponde al número total de palabras $(\mathrm{N})$ evocadas por ambos grupos, para esto se utilizó la prueba t de Student para determinar si existen diferencias significativas en ambos grupos. En este análisis se obtuvo un valor de $(p=0,5)$ lo cual indica que no existen diferencias significativas en lo que corresponde a número de palabras entregadas por los encuestados

promedio de palabras (XR):El segundo estadígrafo, corresponde a los datos arrojados por la prueba t de student para la comprobación de diferencias significativas en el léxico disponible entre hombres y mujeres. En este análisis el valor obtenido corresponde a $(\mathrm{p}=0,6)$ lo cual indica que no hay grandes diferencias entre hombres y mujeres.

número de palabras diferentes (NPD): Este estadígrafo corresponde a los datos arrojados por la prueba t de student, considerando como indicador el número de palabras diferentes (NPD). En el análisis obtenido se puede determinar que dado al valor $(\mathrm{p}=0,7)$ nuevamente se puede demostrar que de acuerdo al número de palabras diferentes, no existen diferencias significativas en el léxico disponible en inglés, considerando la variable género. 
En general, se observa que no existen diferencias significativas en el léxico disponible por ambos grupos, considerando el número total de palabras $(\mathrm{N})$, el promedio de palabras $(\mathrm{XR})$ y el número de palabras diferentes (NPD).

\subsection{Comparativo por centro}

Dado que los resultados obtenidos por la prueba t arrojaron que no existen diferencias significativas en el léxico disponible en inglés como lengua extranjera, se procede a realizar un análisis comparativo para cada centro de interés, con el fin de identificar las diferencias entre los datos entregados por hombres y mujeres, considerando el número de palabras $(\mathrm{N})$, el promedio de palabras $(\mathrm{XR})$, número de palabras diferentes (NPD) y el índice de cohesión (IC), además de un listado de las 10 primeras palabras evocadas por hombres y mujeres para cada centro de interés.

\subsubsection{Centro 1: Animals}

A continuación, se compararán los resultados generales entre hombres y mujeres de la muestra para el centro de interés animals. Realizando una comparación entre hombres y mujeres en el centro de interés animals, se puede observar en la tabla 3 que los hombres escribieron 20 palabras más que las mujeres, obteniendo un promedio de 7.22 palabras, y las mujeres 6.35 palabras en promedio dentro de este centro. Observando el NPD la diferencia de palabras diferentes es solo 4.

TABLA 3

Comparación de IDL entre hombres y mujeres en el centro de interés animals

\begin{tabular}{lllll}
\hline Centros de interés & N & XR & NPD & IC \\
\hline Mujeres & 146 & 6.35 & 24 & 0.26449 \\
Hombres & 166 & 7.22 & 28 & 0.25776 \\
\hline
\end{tabular}

En la tabla 4 se presenta las diez primeras palabras más disponibles, esto es, las 10 primeras palabras que son más evocadas por los alumnos y alumnas.

TABLA 4

Listado de las 10 primeras palabras para la categoría entregadas por Lexmath

\begin{tabular}{llll}
\hline Mujeres & & Hombres & \\
\hline Palabra & IDL & Palabra & IDL \\
CAT & 0,852830435 & DOG & 0,93089087 \\
DOG & 0,838608696 & CAT & 0,836391304 \\
MOUSE & 0,517105463 & MOUSE & 0,476240913 \\
PIG & 0,32282761 & ELEPHANT & 0,362145147 \\
LION & 0,288192217 & PIG & 0,31672888 \\
BEAR & 0,23209814 & BEAR & 0,311321739 \\
TURTLE & 0,220253087 & TIGER & 0,279748147 \\
TIGER & 0,213041826 & TURTLE & 0,257162928 \\
ELEPHANT & 0,210960309 & LION & 0,233582831 \\
MONKEY & 0,202365083 & MONKEY & 0,229104849 \\
\hline
\end{tabular}

Para el centro de interés colors, se puede evidenciar que las primeras diez palabras que mencionan todos los sujetos de la muestra (mujeres y hombres; tabla 4) son las mismas, variando solo el orden en que son 
mencionadas, destacando las tres más disponibles: dog, cat y mouse. Dentro de las palabras más disponibles se encuentra en hombres dog con un IDL de 0,93089087 y en mujeres con IDL de 0,852830435.

\subsubsection{Centro 2: Colors}

A continuación, se comparan los resultados generales entre hombres y mujeres de la muestra para el centro de interés colors. De acuerdo a lo observado en la tabla 5, en el centro de interés colors, el promedio de palabras entregadas por los sujetos en el caso de las mujeres es de 6.73 y de 7.00 en los hombres, presenta una diferencia de 2 palabras diferentes, siendo predominante los hombres con 18 y las mujeres con 16 . Dentro del grado de coincidencia de las palabras entregadas (IC) las mujeres presentan 0.42045 y los hombres 0.38889 .

TABLA 5

Comparación de IDL entre hombres y mujeres en el centro de interés colors

\begin{tabular}{lllll}
\hline Centros de interés & N & XR & NPD & IC \\
\hline Mujeres & 148 & 6.73 & 16 & 0.42045 \\
Hombres & 161 & 7.00 & 18 & 0.38889 \\
\hline
\end{tabular}

En la tabla 6 se presenta las 10 primeras palabras más disponibles en alumnos y alumnas para el centro de interés colors.

TABLA 6

Listado de las 10 primeras palabras para el centro de interés colors

\begin{tabular}{llll}
\hline Mujeres & & Hombres & \\
\hline Palabra & IDL & Palabra & IDL \\
\hline RED & 0,880286364 & RED & 0,939352174 \\
BLUE & 0,739848586 & BLUE & 0,736847748 \\
BLACK & 0,672886596 & BLACK & 0,642192696 \\
PINK & 0,639328738 & GREEN & 0,580080522 \\
GREEN & 0,557457586 & YELLOW & 0,509065083 \\
YELLOW & 0,553568041 & PINK & 0,489718049 \\
ORANGE & 0,264946836 & ORANGE & 0,321886495 \\
PURPLE & 0,213463564 & WHITE & 0,236403673 \\
BROWN & 0,135398496 & PURPLE & 0,228649966 \\
WHITE & 0,093112519 & BROWN & 0,189416387 \\
\hline
\end{tabular}

El centro colors comparte los mismos vocablos, variando el orden en que están disponibles las palabras más comunes para el centro. Las tres palabras más disponibles corresponden a red, blue y black tanto como para mujeres y hombres, variando sólo el IDL que en este caso es mayor el de los hombres. El vocablo red estuvo más disponible para ambos géneros, variando en IDL, siendo 0,939352174 en hombres y 0,880286364 en mujeres.

\subsubsection{Centro 3: Family Members}

Los resultados generales entre hombres y mujeres para el centro de interés family members se presentan en la tabla 7. 
TABLA 7

Comparación de IDL entre hombres y mujeres en el centro de interés family members

\begin{tabular}{lllll}
\hline Centros de interés & N & XR & NPD & IC \\
\hline Mujeres & 85 & 3.86 & 13 & 0.29720 \\
Hombres & 93 & 4.23 & 12 & 0.35227 \\
\hline
\end{tabular}

Family members presenta una diferencia de 8 palabras, obteniendo el menor número las mujeres con 85 palabras y 39 los hombres. El XR presenta una diferencia de 1 palabras entregada por los sujetos, siendo 3.86 en las mujeres y 4.23 en los hombres. El NPD muestra de igual forma una diferencia de 1 palabra por los sujetos, resaltando las mujeres con 13 palabras diferentes y los hombres con 112 palabras.

En la tabla 8 se presenta las diez primeras palabras más disponibles en alumnos y alumnas de la muestra para el centro de interés family members.

TABLA 8

Listado de las 10 primeras palabras para el centro de interés family members

\begin{tabular}{llll}
\hline Mujeres & & Hombres & \\
\hline Palabra & IDL & Palabra & IDL \\
\hline MOM & 0,810863636 & MOM & 0,592272727 \\
GRANDPA & 0,521362727 & GRANDPA & 0,561981364 \\
DAD & 0,501363636 & DAD & 0,530818182 \\
BROTHER & 0,361824136 & BROTHER & 0,358335455 \\
SISTER & 0,334276364 & GRANDMA & 0,308842773 \\
GRANDMA & 0,179145455 & MOTHER & 0,296363636 \\
MOTHER & 0,136363636 & SISTER & 0,290572773 \\
UNCLE & 0,100554546 & FATHER & 0,283590909 \\
GRAND-FATHER & 0,082272727 & UNCLE & 0,103459091 \\
FATHER & 0,081818182 & GRAND-MOTHER & 0,081818182 \\
\hline
\end{tabular}

Family members presenta una gran coincidencia dentro de las primeras 10 palabras entregadas por la muestra, variando en grandfather entregado por mujeres y grandmother entregado por hombres. Dentro de las tres primeras palabras entregadas en este centro se encuentra mom, grandpa y dad para ambos géneros, variando únicamente en el IDL de los encuestados. Mom presenta un IDL de 0,810863636 en mujeres y 0,592272727 , lo que se traduce en que a pesar de ser la más frecuente en ambos géneros, está más disponible en mujeres.

\subsubsection{Centro 4: Food and drink}

Los siguientes son los resultados generales entre hombres y mujeres para el centro de interés food and drink y se presentan en la tabla 9.

TABLA 9

Comparación de IDL entre hombres y mujeres en el centro de interés food and drink

\begin{tabular}{lllll}
\hline Centros de interés & N & XR & NPD & IC \\
\hline Mujeres & 106 & 5.05 & 25 & 0.20190 \\
Hombres & 115 & 5.48 & 26 & 0.21062 \\
\hline
\end{tabular}


Dentro de este centro de interés, se puede establecer que el número de palabras diferentes evocadas por hombres y mujeres es muy similar variando solamente en una palabra más evocada por las mujeres. Sin embargo, cuando se compara el número total de palabras evocadas por los sujetos, los hombres son capaces de nombrar un mayor número de vocablos que las mujeres, siendo un total de 115 en los hombres y 106 en las mujeres.

En la tabla 10 se presenta las diez primeras palabras más disponibles en alumnos y alumnas de la muestra para el centro de interés food and drink.

TABLA 10

Listado de las 10 primeras palabras para el centro de interés food and drink

\begin{tabular}{|l|l|l|l|}
\hline Mujeres & IDL & Hombres & IDL \\
\hline Palabra & 0,598029547 & Palabra & 0,737409048 \\
\hline APPLE & 0,482534259 & APPLE & 0,417651905 \\
\hline ORANGE & 0,324480952 & ORANGE & 0,403620524 \\
\hline KIWI & 0,269151429 & CARROT & 0,365862429 \\
\hline CHEESE & 0,250610143 & CHEESE & 0,287641296 \\
\hline CARROT & 0,244646191 & WATER & 0,284617333 \\
\hline GRAPE & 0,233333333 & GRAPE & 0,273866667 \\
\hline WATER & 0,173425286 & KIWI & 0,255885943 \\
\hline SPAGUETTI & 0,158070952 & ICE-CREAM & 0,14445469 \\
\hline ICE-CREAM & 0,120904762 & SPAGUETTI & 0,133809524 \\
\hline BANANA & PIZZA &
\end{tabular}

Food and drink demuestra palabras similares dentro de las diez primeras, variando en banana entregada por mujeres y pizza entregada por hombres. Las mujeres entregaron como tres primeras palabras fueron apple, orange y kiwi, y los hombres entregaron apple, orange y carrot como las más disponibles. Tanto en el género femenino y masculino la palabra más disponible es apple, variando en un IDL 0,737409048 en hombres y 0,598029547 en mujeres.

\subsubsection{Centro 5: Sports}

Los siguientes son los resultados generales entre hombres y mujeres para el centro de interés sports, de acuerdo a la tabla 11.

TABLA 11

Comparación de IDL entre hombres y mujeres en el centro de interés sports

\begin{tabular}{lllll}
\hline Centros de interés & N & XR & NPD & IC \\
\hline Mujeres & 55 & 2.89 & 11 & 0.26316 \\
Hombres & 83 & 3.61 & 11 & 0.32806 \\
\hline
\end{tabular}

El número total de palabras evocadas por los hombres supera en 28 palabras a las mujeres quienes entregaron 55 palabras. El promedio de palabras (XR) muestra una diferencia de 0.72 palabras, siendo 3.61 en los hombres y 2.89 palabras en las mujeres, a pesar de la diferencia de 1 palabra, es el centro de interés con menor cantidad de vocablos entregados por los sujetos. En el NPD, las palabras diferentes de los sujetos se mantienen sin variación, siendo 11 palabras diferentes para ambos géneros.

En la tabla 12 se presenta las diez primeras palabras más disponibles en alumnos y alumnas de la muestra para el centro de interés sports. 
TABLA 12

Listado de las 10 primeras palabras palabras para el centro de interés sports

\begin{tabular}{llll}
\hline Mujeres & & Hombres & \\
\hline Palabra & IDL & Palabra & IDL \\
\hline TENNIS & 0,626584211 & FOOTBALL & 0,673913044 \\
SOCCER & 0,484210526 & TENNIS & 0,594420435 \\
FOOTBALL & 0,439794737 & BASKETBALL & 0,58126087 \\
BASKETBALL & 0,423631579 & VOLLEYBALL & 0,274156087 \\
VOLLEYBALL & 0,137894737 & SOCCER & 0,267347826 \\
HOCKEY & 0,123631579 & HOCKEY & 0,177495652 \\
GOLF & 0,081 & HANDBALL & 0,163412609 \\
HANDBALL & 0,081 & BASEBALL & 0,134708561 \\
SWIMMING & 0,07371 & GOLF & 0,070826087 \\
BASEBALL & 0,047368421 & AMERICAN-FOOTBALL & 0,054801783 \\
\hline
\end{tabular}

Dentro del centro de interés sports, se aprecian palabras similares tanto para hombres como para mujeres, variando en vocablos como swimming en mujeres y American football en hombres. Se observa, que la palabra más disponible para las mujeres es tennis con un IDL de 0,626584211, y en hombres la palabra más disponible es football con un IDL de 0,673913044 .

\section{Discusión Y CONCLUSIONES}

El presente estudio tuvo como objetivo analizar cuantitativamente el efecto del factor género en la disponibilidad léxica en inglés como lengua extranjera en una muestra de 46 estudiantes de quinto año básico de un colegio de administración subvencionada.

Los datos cuantitativos muestran que en términos de número total de palabras $(\mathrm{N})$ los hombres producen 123,6 palabras y las mujeres 108. En el promedio de palabras (XR), el género masculino logra evocar un número de 5,50 palabras y el género femenino logra evocar 4,97. Finalmente cuando se observa el número de palabras diferentes (NPD), los hombres producen 19 palabras y las mujeres logran producir 17.8 vocablos diferentes. Lo que demuestra que en cada centro de interés existe un predominio del género masculino. Sin embargo, al realizar una prueba t de Student con los datos previamente recogidos, se observa que no existen diferencias significativas en el léxico de los hablantes. Este resultado concuerda con los estudios previamente realizados en español como lengua materna por Pacheco et al. (2017) y Cepeda et al. (2014), en los cuales se puede observar que la variable "género" no tiene una incidencia en el léxico disponible de los hablantes. Sin embargo, no concuerdan con los resultados obtenidos en pruebas de disponibilidad léxica en inglés como lengua extranjera, en los cuales se observa que las mujeres superan a los hombres en la producción léxica (Jiménez y Ojeda, 2009; Fernández, 2010; Agustín y Fernández, 2014).

$\mathrm{Al}$ realizar un análisis más exhaustivo de los datos obtenidos se observa lo siguiente:

- La variable género no produce una gran incidencia dentro de los índices de la disponibilidad léxica de los sujetos encuestados (Pacheco et al., 2017).

En términos cuantitativos, si existe una diferencia entre el léxico disponible que tienen hombres y mujeres, la que, sin embargo, no logra ser significativa.

Existe un predominio del género masculino en cada uno de los centros de interés y considerando los índices de N, XR y NPD, este último teniendo un predominio femenino, produciendo una palabra más que los hombres.

Para el centro de interés animals, el género masculino destaca en todos los índices de medición, produciendo un mayor número de palabras, promedio de palabras y número de palabras diferentes. 
El género femenino, predomina dentro del centro de interés colors, obteniendo altas cifras en cada uno de los índices de medición

Por su parte, la descripción cualitativa de las 10 primeras palabras entregadas por el grupo de hombres y mujeres

en cada centro de interés, muestra que las 10 primeras palabras entregadas por ambos grupos tienden a ser las mismas en todos los centros de interés estudiados, presentando algunas leves variaciones en la disponibilidad de las mismas. En general, estas listas se diferencian en el grado de disponibilidad léxica de los vocablos, lo que muestra que a pesar de entregar las mismas palabas en cada centro de interés, la disponibilidad de ellas tendería a variar entre hombres y mujeres (Sandu, 2013).

Esta investigación ha dado luces con respecto a la variable género y al léxico disponible en inglés como lengua extranjera. Sin embargo, el trabajo realizado presenta las siguientes limitaciones: por un lado, el tamaño de la muestra, el cual al ser pequeño no es representativo para todos los alumnos de quinto año básico, y, por otro lado, lo limitado de los centros de interés incluidos en la investigación.

A raíz de estas limitaciones, se requiere una mayor investigación en esta área, considerando muestras más grandes y además aplicándose a distintos niveles de educación teniendo en consideración los ítems léxicos (centro de interés) abordados en cada uno de los niveles, siguiendo las pautas entregadas por los planes y programas del Ministerio de Educación. Teniendo en cuenta estos criterios, el estudio en léxico disponible puede constituir un aporte a la enseñanza de lenguas extranjeras para favorecer el aprendizaje léxico del idioma inglés en educación básica, y a la vez, constituir un elemento que permita conocer los cambios que ocurren en el lexicón mental de los estudiantes en las distintas etapas de su proceso educativo, con el fin de tomar acciones remediales que favorezcan la adquisición léxica en lengua extranjera.

\section{ReFERENCiAS}

Agustín, M. y Fernández, A. (2014). Lexical Variation in Learners' Responses to Cue Words: The Effect of Gender. En Jiménez Catalan, R. (Ed.) Lexical Availability in English and Spanish. Berlin: Springer.

Agustín-Llach, M. \& Terrazas, M. (2012). Vocabulary Knowledge Development and Gender Differences in a Second Language. Estudios de Lingüística Inglesa Aplicada ELIA, 12, 45-75. Recuperado de http://revistas.uned.es/ index.php/ELIA/article/view/18027

Areta, M. (2009). El léxico disponible de los estudiantespreuniversitarios navarros. Estudio sociolingüistico [Tesis doctoral no publicada]. Universidad de Navarra, Pamplona.

Calero, M. y Serrano, M. (2019). Incidencia del factor sexo en el léxico disponible de una comunidad bilingüe. Ogigia. Revista electrónica de estudios hispánicos, 25, 83-107. https://doi.org/10.24197/ogigia.25.2019.83-107

Cepeda, M.; Granada, M. y Pomes, M. (2014). Disponibilidad léxica en estudiantes de primero básico. Literatura y linguistica, 30, 166-181. http://dx.doi.org/10.4067/S0716-58112014000200010

Fernández, A. (2010). Gender and Motivation in EFL Vocabulary Production. En Jiménez Catalán (ed.) Gender Perspectives on Vocabulary in Foreign Languages (pp. 93-116). London: Palgrave Macmillan.

Galdames, A.; Guerrero, S. y Toledo, G. (2018). Disponibilidad léxica de aprendientes de español como segunda lengua en Santiago de Chile: una plataforma para la enseñanza del léxico. Logos: Revista de Lingüistica, Filosofía $y$ Literatura, 28(1), 135-150. http://dx.doi.org/10.15443/rl2811

Galloso, M. (2003). El léxico de los estudiantes preuniversitarios en el distrito universitario de Salamanca (Ávila, Salamanca y Zamora). Burgos: Fundación Instituto Castellano y Leonés de la Lengua.

Garzón Segura, A. y Penagos López, L. (2016). Disponibilidad léxica en estudiantes de primer semestre de pregrado de una institución universitaria de Villavicencio, Colombia. Forma y Función, 29(2), 63-84. http:// dx.doi.org/10.15446/fyf.v29n2.60189 
González, A., y Orellana, P. (1999). Análisis del comportamiento de la variable sexo en el léxico disponible de Cádiz. REALE, (11), 65-73.

Gougenheim, G., Michéa, R., Rivenc, P. \& Sauvageot, A. (1956). L'élaboration du francais élémentaire. Paris: Didier.

Hernández, N. (2006). Hacia una teoría integrada de la disponibilidad léxica: el léxico disponible de los estudiantes castellanos-manchegos. Salamanca: Ediciones de la Universidad de Salamanca.

Hernández, N. y Tomé, C. (2017). Léxico disponible en primera y segunda lengua: bases cognitivas. En De La Rosa, F. (ed.). Palabras vocabulario léxico: la lexicología aplicada a la didáctica y a la diacronia (pp. 99-122). Venezia: Edizioni Ca' Foscari.

Jiménez, R. \& Ojeda, J. (2009). Girls'and Boys'Lexical Availability in EFL. ITL, International Journal of Applied Linguistics, 158, 57-76.

Lightbown, P. y Spada, N. (2013). How Languages Are Learned (4th Ed.). Oxford, New York:Oxford University Press. Lorán, R. \& López, H. (1983). Nouveau Calcul de l'Indice de Disponibilité. Universidad de Puerto Rico.

Michéa, R. (1953). Mots fréquents et mots disponibles. Un aspect nouveau de la statistique du langage. Les langues modernes, 47, 338-344.

Pacheco, C., Cabrera, J. y González, I. (2017). Incidencia de la variable sexo en la disponibilidad léxica de estudiantes de preuniversitario en Pinar del Río, Cuba. Íkala, Revista de Lenguaje y Cultura, 22(2), 237-253. http:// dx.doi.org/10.17533/udea.ikala.v22n02a05

Palapanidi, K. (2012). La aplicación de la disponibilidad léxica a la didáctica del léxico de LE. Revista Nebrija de Lingüistica Aplicada, 6(11), 62-70. https://doi.org/10.26378/rnlael611176

Payne, C. (2016). Lexical availability. English teaching professional, 102, 18-20.

Quintanilla, A. y Salcedo, P. (2019). Disponibilidad léxica en procesos de formación inicial de futuros profesores de inglés. Revista Brasileira de Linguistica Aplicada, 19(3), 529-554. https:// dx.doi.org/10.1590/1984-6398201913157

Sandu, B. (2013). Romanian Students' Lexical Availability in Spanish as a Foreign Language. Estudios interlingüisticos, $1,121-133$.

Trigo, E. y González, A. (2011). Estudio del comportamiento de la variable sexo en el léxico disponible de los preuniversitarios sevillanos. Diálogo de la lengua, 3, 28-41.

Verdeses-Mirabal, R. (2012). Disponibilidad léxica de los estudiantes hispanos de Redwood City, California. Revista Nebrija de Lingüistica Aplicada a la Enseñanza de las Lenguas, 6(11), 6-52. https://doi.org/10.26378/ rnlael611173 